\title{
Cannabinoide in der Schmerzmedizin
}

\section{Ist eine Liberalisierung von medizinischem Cannabis sinnvoll?}

\author{
Cannabinoide sind für viele Ärzte unverzichtbar für Patienten, für die keine hinreichend wirksamen Arznei- \\ mittel zur Verfügung stehen. Doch wie sieht es mit dem gesicherten Nachweis der analgetischen Wirkung \\ von Cannabinoiden in placebokontrollierten Studien aus? Eine Debatte.
}

\section{Pro: Cannabinoide können die Lebensqualität verbessern}

W ir am Universitätsklinikum des Saarlandes Homburg/Saar versorgen bereits seit mehr als einem Jahrzehnt Patienten ab dem Säuglingsalter mit cannabinoidhaltigen Rezepturen für verschiedenste Indikationen, auch fernab von Schmerz (etwa Spastik, unbeherrschbare Unruhezustände bei schwerst Mehrfachbehinderten). Dabei haben wir auch in der Langzeitanwendung bei Kindern und Jugendlichen weder die Notwendigkeit für Dosiseskalationen beobachtet, noch relevante psychotrope Effekte oder gar Persönlichkeitsveränderungen gesehen.

Cannabinoide sind aus unserer Sicht eine anerkannt wirksame Therapieoption in der Palliativversorgung und der Versorgung multimorbider hochkomplexer Patientenkollektive, für die keine hinreichend wirksamen Fertigarzneimittel zur Verfügung stehen. Der Cannabiswirkstoff Dronabinol wirkt appetitanregend, muskelrelaxierend, brechreiz- und schmerzlindernd, angstlösend und entzündungshemmend. Mit dieser Wirkweise kann Dronabinol maßgeblich die Lebensqualität von Palliativ- oder Chemotherapiepatienten verbessern und ist ein wesentlicher Baustein bei der Versorgung schwerst mehrfachbehinderter Kinder. Die Erstattungssituation für Dronabinol ist jedoch nach wie vor unzureichend. Daher begrüßen wir den im Januar veröffentlichten Referentenentwurf der Bundesregierung, der eine leichtere Erstat- tung von Cannabinoiden beabsichtigt [1]. Diese Initiative ist umso wichtiger, da es sich bei der gesamten Substanzklasse um eine zwar vielversprechende, aber immer noch stigmatisierte und aufgrund der mangelnden Patentierbarkeit von natürlichen Wirkstoffen für die Industrie wenig attraktive Forschungsplattform handelt. Das erklärt eventuell auch, dass die Studienlage nur für das Fertigarzneimittel Sativex ${ }^{\circledast}$, das aus Dronabinol und Cannabidiol besteht und Dronabinol, das in den USA als Marinol ${ }^{\circledR}$ für verschieden Indikationen zugelassen ist, als relativ gut gesichert gilt. Für den Bereich der Pädiatrie und auch bei vielen geriatrischen $\mathrm{Pa}$ tienten ist das große Applikationsspektrum von Rezepturen enorm wichtig. So können wir individuell entscheiden, ob der Patient das Dronabinol als ölige Tropfen, Kapseln, Inhalat oder Sondennahrung erhält, das ist gerade bei unserem schwerstkranken und heterogenen Patientenkollektiv von großem Vorteil. Auch die individuellen Dosierungen stellen für die Apotheker kein Problem dar.

Letztlich ist es aber bittere Realität, dass in der Pädiatrie bis zu $80 \%$ und in der Palliativversorgung bis zu $50 \%$ Offlabel-use üblich sind, und immer schwingt das Regressrisiko mit.

Wissenschaftliche Erkenntnisse zu teilweise unzureichend definierten Extrakten oder zu „medizinischem Cannabis“ besitzen meist nur anekdotischen Charakter und keine ausreichende Evidenz.
Die Bundesärztekammer hat in Ihrer Stellungnahme zum Referentenentwurf betont, dass die wissenschaftliche Datenlage für standardisierte und in kontrollierter Dosis einsetzbare Cannabis-Arzneien für bestimmte Anwendungsgebiete für eine Verordnung zu Lasten der gesetzlichen Krankenversicherung (GKV) ausreichend sei [2]. In der BÄK stoßen die Pläne, auch die Kostenübernahme von getrockneten Cannabis-Blüten und Extrakten zu ermöglichen, auf strikte Ablehnung, da es im Gegensatz zu cannabinoidhaltigen Rezeptur- und Fertigarzneien für deren medizinischen Einsatz an ausreichender wissenschaftlicher Evidenz fehlt. Zudem ist zu berücksichtigen, dass der Gebrauch von Medizinalhanf keine genaue Dosierung der medizinisch wirksamen Komponenten von Cannabis erlaubt und der Gebrauch als Joint mit den gesundheitlichen Gefahren des Tabakrauchens verbunden ist.

\section{Literatur}

1. www.bmg.bund.de/fileadmin/dateien/ Downloads/Gesetze_und_Verordnungen/ GuV/C/160108_GE_Cannabis_als_Medizin mit_Cannabisagentur.pdf

2. www.bundesaerztekammer.de/presse/pressemitteilungen/news-detail/baek-unterstuetzt-kostenuebernahme-fuer-cannabis-fertigarzneien-und-rezepturen/

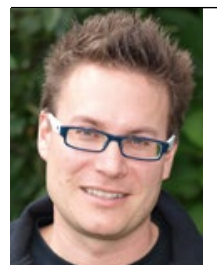

Prof. Dr. med. Sven Gottschling Zentrum für Palliativmedizin und Kinderschmerztherapie Universitätsklinikum des Saarlandes 